\title{
HEALTH-ORIENTED EVALUATION OF THE SPATIAL DISTRIBUTION OF URBAN GREEN SPACE IN THE WUHAN INNER CITY AREA OF CHINA
}

\author{
YUPING DONG ${ }^{1,2} \&$ HELIN LIU ${ }^{1,2}$ \\ ${ }^{1}$ School of Architecture and Urban Planning, Huazhong University of Science and Technology, China \\ ${ }^{2}$ Centre for Urban and Rural Planning Support Research, Huazhong University of Science and Technology, China
}

\begin{abstract}
Research has proved that urban green space can be utilized to promote public health. While exploring the underlying mechanism, the main focus is always on the meso-scale characteristics of urban green space such as accessibility, availability, proximity, and its micro-scale attributes like area, quantity, constituent elements and environmental perception. Yet, its spatial distribution, as the macro-scale characteristics of urban green space, is also closely related to residents' health but has not well been explored. Regarding this, this paper takes the inner-city area of Wuhan as an example to evaluate the spatial distribution of the green space with Jiedao as the basic analysis unit. By buffer analysis and zonal analysis, the evaluation is conducted from five dimensions which are balance, equality, availability, accessibility and quality. The results show that, except in balance, the distribution of health-promotion green space is not satisfactory in the other four dimensions, especially in accessibility, quality and equality. The underlying causes could be attributed to three aspects of the current urban green space planning: (1) emphasis on ecology-oriented value and the application of "top-down" planning approach; (2) adoption of a single-dimension index system; (3) application of a static but not an adaptive framework. Therefore, from planning perspective, to shift the value-orientation and readjust the framework of urban green space planning will be one key solution to the identified problems.

Keywords: health-oriented, urban green space, evaluation, planning, Wuhan inner city area.
\end{abstract}

\section{INTRODUCTION}

Substantial studies have proved that urban green space can promote public health through both regulating ecosystem services (such as improving air quality, relieving heat island effect) and cultural ecosystem services (such as improving physical activity, reducing stress) [1]. Despite this fact, it is still common to see green space land being squeezed by other land uses during the urbanization processes. As a result, on the one hand it leads to unequal accessibility and availability of health-related benefits from urban green space [2]; on the other hand, it causes the planning and management of green space in urban area a challenge [3]. What's more, the prevalence of sedentary life style and high stressful living environment deteriorates residents' sub-health status [4], [5]. Therefore, it is significant to examine the spatial distribution of urban green space from health-promotion perspective so as to facilitate exploration of planning solutions to the identified problems.

However, on this topic most studies in China tend to focus on landscape appreciation and eco-value rather than equality and accessibility [6], [7]. Hu et al. [8] insisted that the existence of green space does not means it can be physically or socio-economically accessed by residents. Even if those dimensions are counted, the focus is on the supply of the resource itself but not its health effect to users [9], [10]. In other words, those studies are more likely to study availability rather than accessibility. That is, the principle is space-oriented rather than user-oriented. Moreover, from the health-outcome perspective, it can be identified that good availability does not mean that the green spaces can function well in terms of healthpromotion. For example, Hystad et al. [11] find that birth outcomes have a positive and linear relation with residential greenness level when the NDVI (Normalized Differential Vegetation 
Index) value is or greater than 0.15. Similarly, Markevych et al. [12] get the results that low and moderate greenness level can lead to higher diastolic blood pressure for children, compared with high greenness level.

So, this paper aims to evaluate the spatial distribution of urban green space by integrating the health-promotion dimension. Firstly, it summarizes the indicators that can describe green space's potential to promote public health. Then we apply those indicators to evaluate the green space of Wuhan inner city from the perspectives of balance, equality, availability, accessibility and quality which are related to health-promotion. By referring to current distribution problems identified, the underlying causes are then discussed, and the correspondent solutions are proposed in the view of urban green space planning.

\section{INDICATORS TO DESCRIBE HEALTH-ORIENTED CHARACTERISTICS OF URBAN GREEN SPACE}

A series of research on the relationship between urban green space and health outcomes have adopted different indicators to measure users' exposure to green space, which can be combed into three categories, namely, accessibility, availability and self-feature. In detail, accessibility pays more attention to individual's actual acquirement and usage of urban green space with consideration of equality; availability focuses on the provision and distribution of but not residents' real engagement with the urban green space; self-feature concentrates on the nature of urban green space that could make difference in cultural ecosystem services.

The mostly adopted indicators to describe accessibility are as follows: NDVI and percentage of green space (PGS) within a buffer distance from a certain point (usually residents' home), distance from residents' home to a specific green space and residents' assessment/perception of accessibility to green space. For example, by measuring the PGS within the radius of 1 and $3 \mathrm{~km}$ around individual addresses respectively, Maas et al. [13] find that this indicator in both buffer distances has positive correlation with respondents' perceived health-benefits no matter what the urbanization degree is. By the same indicators, Maas et al. [14] find in another study that there is a significant relationship between accessibility and respondents' loneliness feeling. In other words, the greener the residents' living environment is the better accessibility and less loneliness they may have. They also report that increase of the PGS within a buffer of $1 \mathrm{~km}$ can reduce the feeling of lacking social support. Storgaard et al. [15] even prove that if the PGS within a 1 and $2.5 \mathrm{~km}$ buffer distance is lower than $5.4 \%$ and $8.6 \%$ respectively, residents would be more likely to have sedentary behavior compared with the situation that the PGS is greater than $20 \%$.

From the perspective of availability, most studies use indicators as below: NDVI and PGS within a certain area, percentage of a specific green space (such as the proportion of park or public open space) in and assessed/perceived greenness of a certain area. For example, Almanza et al. [16] conclude that NDVI has a positive association with the rate of MVPA (moderate-to-vigorous physical activity). That is to say that high greenness level could promote MVPA. This finding aligns with a dose-response study derived from GrigsbyToussaint et al. [17], in which greenness level (measured by NDVI) is proved to be the significant factor to influence preschool children's time for outdoor physical activity. Specifically, one unit increase of NDVI correspondingly can lead to an increase of about a 3-minute outdoor physical activity.

Of the many indicators to capture green space's self-feature, most are natural elements, types of available facilities, aesthetics/attractiveness, safety, general perception of quality and organized/programmed activities. For example, vegetation is considered as a vital natural element to improve environmental aesthetic, which contributes to attract more users and enhance their satisfaction [18]. Therefore, the perception of vegetation quality, such as its 
structure and diversity, could lead to difference in the usage of urban green space for outdoor physical activities [19]. What's more, according to the survey conducted by Schipperijn et al. [20], the natural elements like trees and water are the respondents' most favorite features which significantly increase their engagement in physical activities. More precisely, by a meta-analysis Barton and Pretty [21] find that improvement of self-esteem and mood increases by 0.29 and 0.19 respectively if there is water element in the green space.

The above review implies that: (1) most research focus on a relatively small-scale area, such as neighborhood, rather than the whole city to study health-related benefits by considering spatial equality. So those findings can only provide reference criteria for detailed planning of a certain site but not the overall layout of green space within a city for public health, an issue widely overlooked in planning practice; (2) health-oriented characteristics of urban green space are diverse, which means that a variety of indicators are needed to describe them. In addition, both quantitative and qualitative indicators are crucial for precise description of green space's potential to generate health benefits. Therefore, it is necessary to take different categories of indicators (including accessibility, availability and self-feature) into consideration while conducting health-oriented evaluation of the distribution of urban green space; (3) one characteristic of urban green space could generate more than one healthrelated benefit. For example, the PGS within a $1 \mathrm{~km}$ buffer can contribute to general perceived health, loneliness feeling, social support, and physical activity level etc.

\section{METHODOLOGY}

\subsection{Study area}

Wuhan is the capital city of Hubei Province, located in the middle of China and the middle reaches of the Yangtze River. As one of China's megalopolises, in 2016 its urbanization rate is $79.77 \%$ and its GDP per capita is 111,469 Yuan. Wuhan covers $8,569.15 \mathrm{~km}^{2}$ and consists of 13 districts (a district is a kind of administrative division level in China's cities. Each district in general consists of several Jiedaos and Jiedao is the lowest administrative level in cities in China). This research will focus on the inner-city area which consists of 7 districts (Jiang'an, Jianghan, Qiaokou, Hanyang, Wuchang, Qingshan and Hongshan) including 96 Jiedaos (including East Lake High-Tech Development Zone, East Lake Ecotourism Scenic Zone and Wuhan Chemical Industry Park) with a total area of $955.15 \mathrm{~km}^{2}$ (Fig. 1).

In Wuhan, it is reported that residents' engagement in physical activity is of low frequency, usually less than 3 days a week [22]. In 2015, only about 480.2 thousand people take regular physical activity in Wuhan [23], which means that around $95 \%$ of the residents live a sedentary lifestyle. What's more, the space suitable for sport is insufficient as the sport field area is $1.43 \mathrm{~m}^{2}$ per capita [24], lower than 1.57, the average level in China [25].

\subsection{Study design}

In this study, urban green space refers to all kinds of green land use within the boundary of Wuhan inner city. In addition, water (lake and river, etc.) is also treated as urban green space by considering two points: first, as a natural element, it functions like green space and can exaggerate or even directly generate health-related benefits; second, of the inner city area approximately one fifth is covered by water (The area of water body is extracted from remote sensing image). Considering data availability, we take Jiedao as the study unit. The vector Jiedao map with geo-locations and population in 2014 is offered by Wuhan Statistics Bureau. 


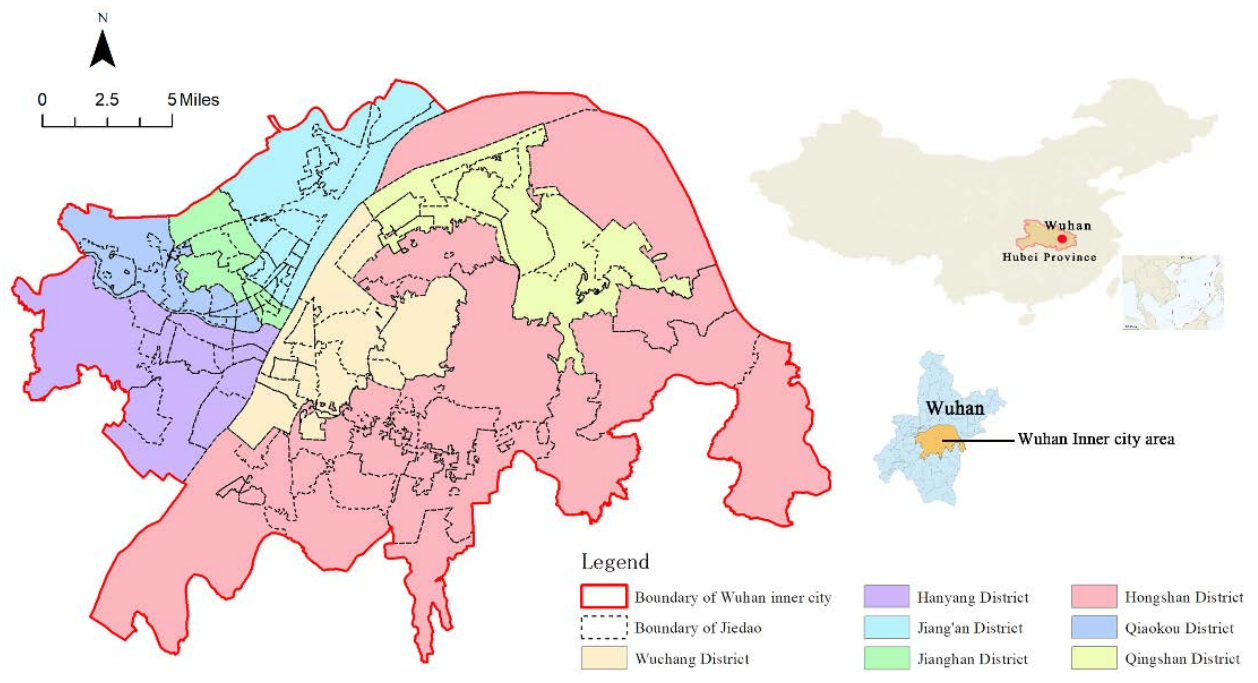

Figure 1: The location and the Jiedaos of Wuhan Inner city area

Series of studies have proved that characteristics of the spatial distribution of urban green space, like the distance (to/from a certain site), NDVI, PGS, etc., have a significant influence upon the level of outdoor physical activity. And epidemiological findings have revealed that regular physical activity could prevent chronic diseases and premature death [26]. Hence, for public health purpose it is meaningful to focus on the spatial distribution of urban green space, which may help to alleviate the threat from the non-communicable diseases that have been reported as the major risk to public health in Wuhan [22].

Firstly, we use concentration index and Gini Coefficient with the goal to identify the spatial distribution characteristics from a macro perspective. Then we evaluate the availability, accessibility and quality of urban green space by taking Jiedao as the analysis unit. Indicators adopted to describe those three aspects are NDVI, the percentage of urban green space within $1 \mathrm{~km}$ buffer distance and the ratio of arbor to shrub proposed by Zhu and $\mathrm{Wu}$ [27] and Zhang et al. [28], respectively.

\subsection{Explanation of indicators}

\subsubsection{Concentration index}

Concentration index is usually applied to measure the spatial concentration degree of certain resource. The equation is presented below and detailed explanation of it can be found in Rui et al. [29] and Zhu et al. [30].

$$
\mathrm{C}=\left(\sqrt{\sum_{i=1}^{n}\left(H_{i} / S\right)^{2}} / \sqrt{\sum_{i=1}^{n}(1 / n)^{2}}\right)-1,
$$

where $H_{i}$ represents the area of green space (including water body) within Jiedao $i$; $S$ represents the total area of green space (including water body) within Wuhan inner city area; and $n$ represents the total number of Jiedao within Wuhan inner city area (it is 96 in our study). 


\subsubsection{Gini Coefficient}

Gini Coefficient is an index proposed by Gini, the Italian economist, in 1922 to quantitatively measure the income gap among different groups. Nowadays, it has evolved into many revised forms in different fields. In this paper we adopt what $\mathrm{Wu}$ [31] has proposed. When the area density (It refers to the percentage of green space within Jiedao unit) of green space within every Jiedao unit is calculated and ranked in an ascending order, then the Gini Coefficient $G$ can be calculated by the eqns (2)-(4):

$$
\mathrm{G}=\sum_{i=1}^{n-1}\left(F_{i} * R_{i+1}-F_{i+1} * R_{i}\right),
$$

where

$$
\begin{aligned}
& F_{i}=\sum_{k=1}^{i}\left(P_{k} / P\right), \\
& R_{i}=\sum_{k=1}^{i}\left(H_{k} / S\right),
\end{aligned}
$$

where $P_{k}$ represents the number of residents within Jiedao $k ; P$ represents the total number of residents within Wuhan inner city area; $H_{k}$ represents the area of green space (including water body) within Jiedao $k$; $S$ represents the total area of green space (including water body) within Wuhan inner city area; $n$ represents the total number of Jiedao within Wuhan inner city area (it is 96 in our study).

\subsubsection{NDVI}

NDVI is usually applied to measure the greenness of residential environment, which has a strong association with perception of greenness, urban green space usage, physical activity, mental health outcome, etc. The value of NDVI is derived from near-infrared (NIR) bands and visible red (RED) bands of remote sensing image and it ranges from -1(usually regarded as water) to +1 (usually regarded as lush vegetation coverage). In this study, we obtain the NDVI values with a resolution of $30 \mathrm{~m} \times 30 \mathrm{~m}$ based on the sensing map from Landsat8 (OLI_TIRS) in July 23th 2016. The mean NDVI value for each Jiedao is calculated by zonal analysis supported by spatial analyst tools in Arcgis 10.2.

\subsubsection{Percentage of green space (PGS)}

The PGS within a certain buffer distance is usually applied to measure the accessibility of urban green space [32]. In this study, the buffer distance around the centroid of Jiedao is defined to be $1 \mathrm{~km}$ (straight-line) which is commonly used in research on the health outcomes of urban green space [14]. The centroid of each Jiedao is treated as the clustering point of population and its coordinate is the default XYs in Arcgis 10.2.

\subsubsection{The ratio of arbor to shrub}

High accessibility does not mean high usage if urban green space is of poor quality in aesthetics and design. Therefore, it is significant to consider urban green space's quality for health-oriented evaluation of its distribution. For this paper we use the ratio of arbor to shrub proposed by Zhang et al. [28] as the indicator. The vegetation types (namely, grass, bush and tree) is classified based on the suggested NDVI value intervals [33] and the local context in Wuhan. Then for each Jiedao, the area of trees and for bushes within the one-kilometer buffer are extracted respectively by zonal analysis with Arcgis 10.2, which provide the required data for the calculation of the ratio of arbor to shrub. 


\section{RESULTS OF THE EVALUATION AND THE UNDERLYING CAUSES}

\subsection{Results of the evaluation}

From eqn (1), it is calculated that the concentration index of the current urban green spaces approximates to 0.86 . If the green spaces are evenly distributed in the 96 Jiedaos, the corresponding index value is 0 ; and if all the green spaces are in the largest Jiedao, then we have the maximum value for the concentration index which is 4.6. So, regarding the value of 0.86 , we can conclude that without considering demographic elements the spatial distribution of the green space in Wuhan inner city are is relatively even.

Yet, if we count in the people that the green space has to serve in each Jiedao, then we will see that the spatial distribution of green space in Wuhan inner city area is seriously unfair as the Gini Coefficient is around 0.695, much higher than 0.5, a value meaning seriously unfair according to general criteria [31].

Based on the NDVI map (Fig. 2), we can generate the detailed NDVI distribution map at Jiedao-level (Fig. 3) by zonal analysis. It can be seen that, although the greenness level is high in the whole inner-city area, the NDVI values are relatively low for the Jiedaos and most of them are between 0.15 and 0.45 , especially in the interval of $[0.25,0.35]$. What's more, Jiedaos next to the suburban areas have a relatively higher greenness level while the ones in the very central area have a lower level, even with some Jiedaos having a value lower than 0.15. According to findings by Hystad et al. [11], it means that the current greens in these Jiedaos cannot function well to generate significant benefits for pregnancy and birth.

As explained, water to some extent can function like green space to attract people to take physical activities around. But people cannot wander in water like what they can do on grassland and in woods. Regarding this difference, the accessibility of urban green space is calculated by PGS in two scenarios: one with water included and the other with water excluded. The results are depicted by Figs 4 and 5, respectively. It is evident that, in terms of health-promotion, most Jiedaos have poor accessibility to urban green space, especially those Jiedaos in the central part. Even if water is counted in, we can still see that Jiedaos in Jianghan, Qiaokou and Hanyang districts have very lower greenness accessibility.

After zonal analysis, the ratios of arbor to shrub for all the Jiedaos are shown in Fig. 6. According to Zhang et al. [28], when the ratio of arbor to shrub approximates 1:1.5 (namely, 0.667 ), to user's feeling the aesthetic quality of urban green space can be better. So, the results (Fig. 6) imply that the aesthetic quality in most Jiedaos still have much space to improve. And if we compare it with Fig. 5, we can see that many Jiedaos have a low quality of urban green space even though they enjoy relatively high accessibility.

According to the results above, we can see that the spatial distribution of urban green space in the inner-city area of Wuhan is well balanced among different Jiedaos in a general sense. Yet, if we consider the residents that each Jiedao has to serve, then it is evident that most Jiedaos, especially those in the central part of the inner-city area have a relatively low availability and accessibility, even if water is treated as green space. In addition, the aesthetic quality of the green space is also not satisfactory in most of the Jiedaos, particularly those once specializing in traditional heavy industries and old transport complex.

\subsection{The underlying causes from planning perspective}

The identified problems above may result from many causes, such as the bequeathed builtup environment based upon overemphasis on work rather than leisure in urban life or overlook of the quality of public open space in cities in the second half of the 20th century 


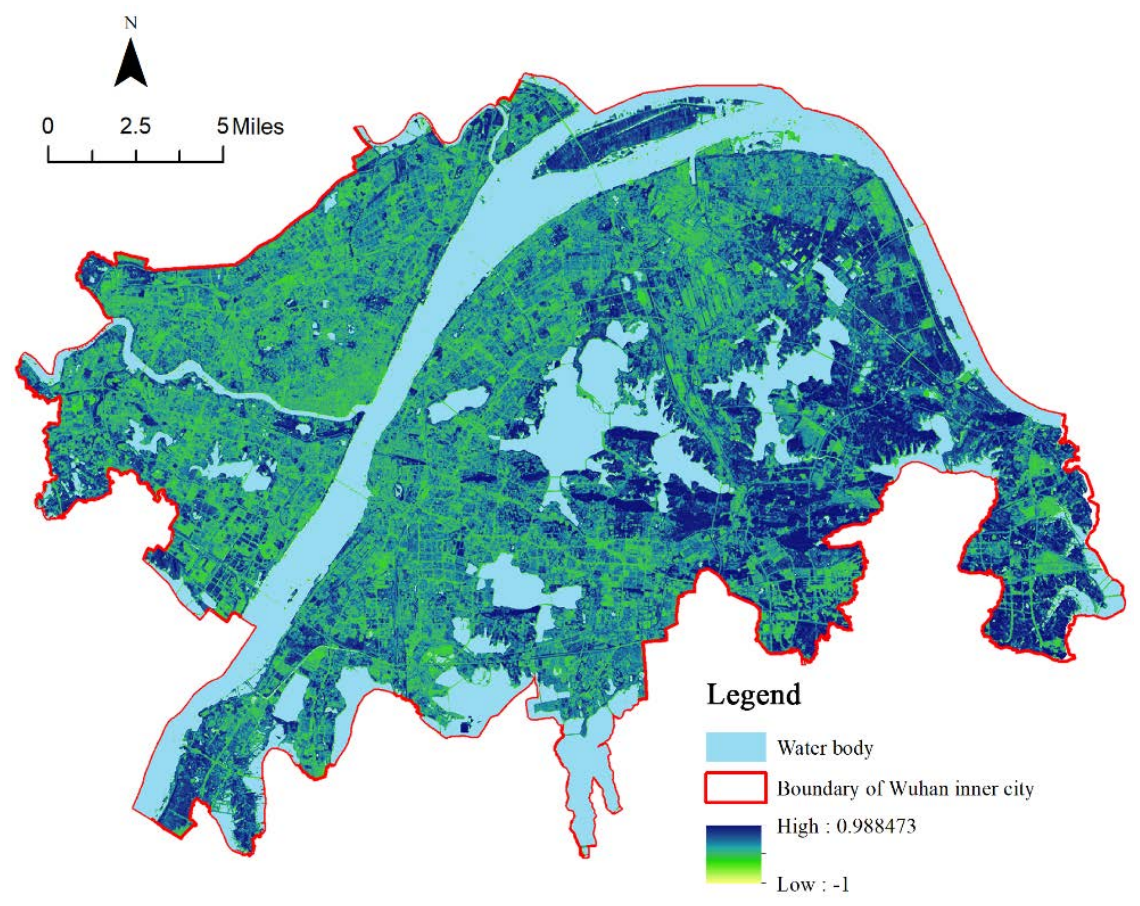

Figure 2: The NDVI of Wuhan inner city area.

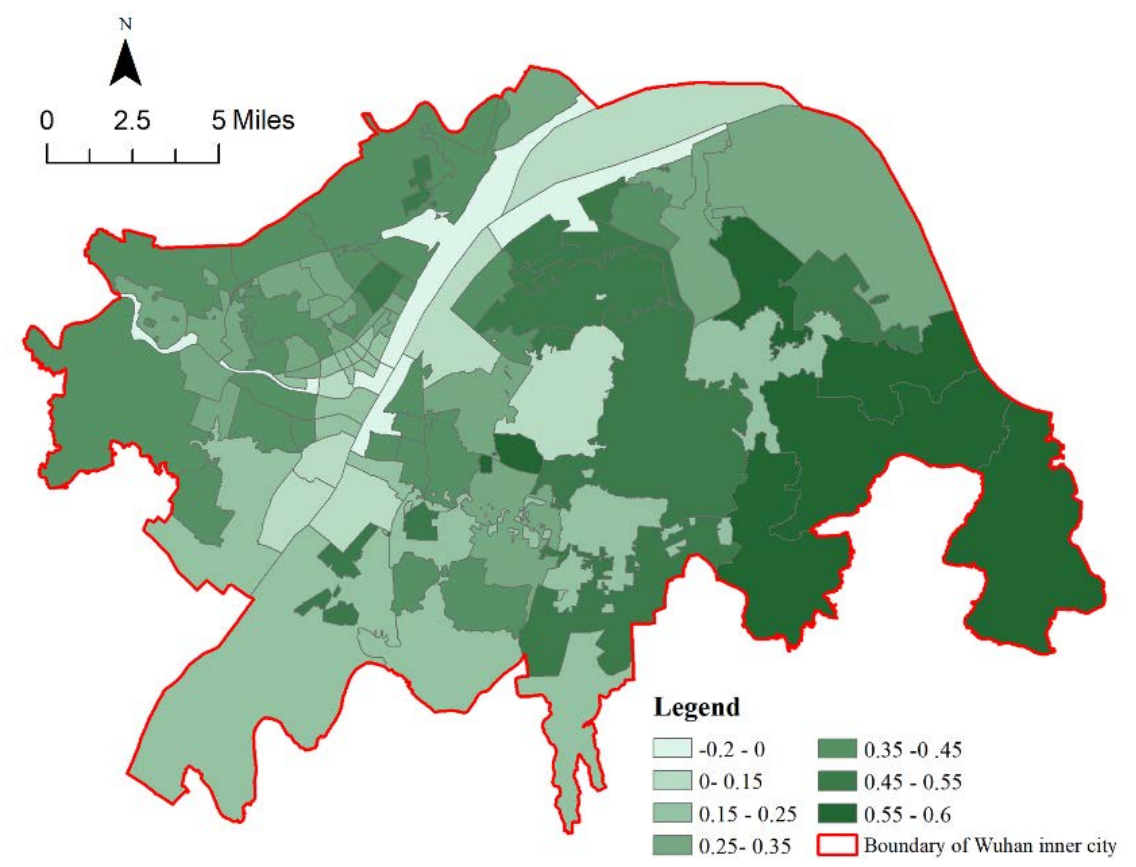

Figure 3: The NDVI distribution at Jiedao-level. 


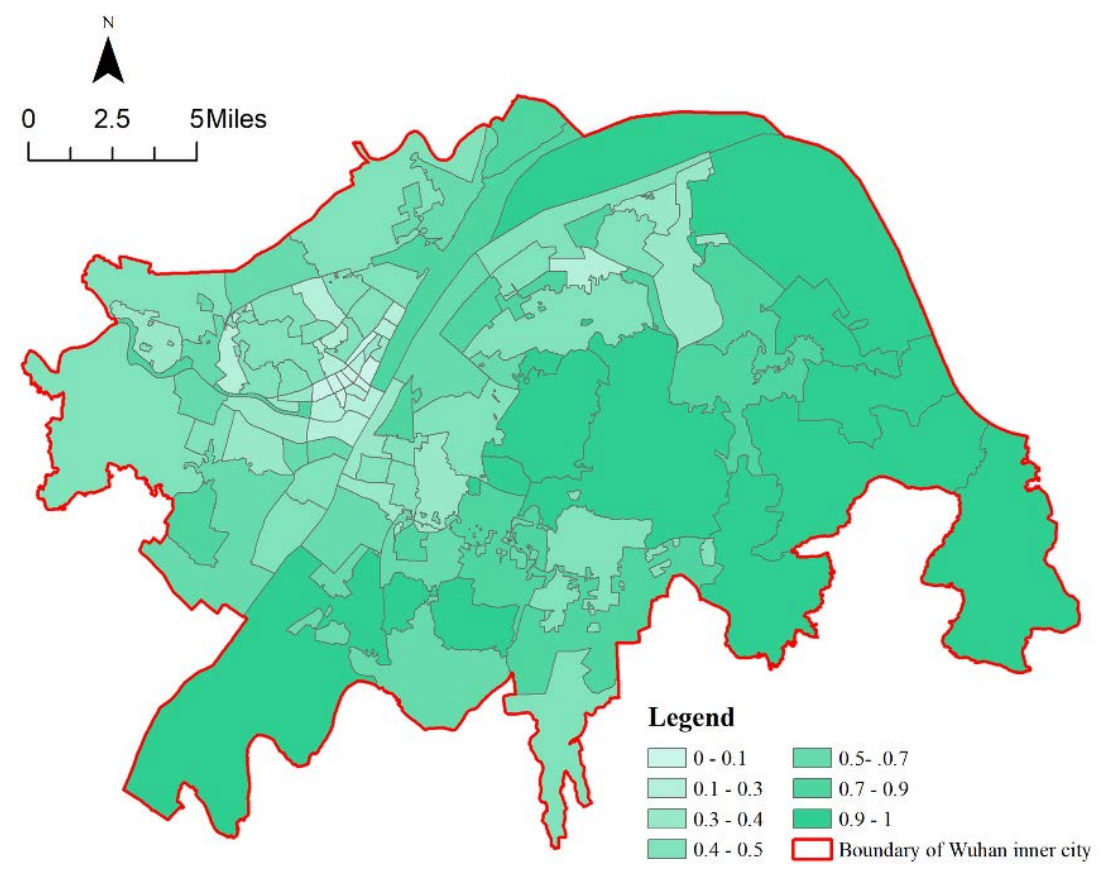

Figure 4: The percentage of urban green space within a buffer distance of $1 \mathrm{~km}$ (with water).

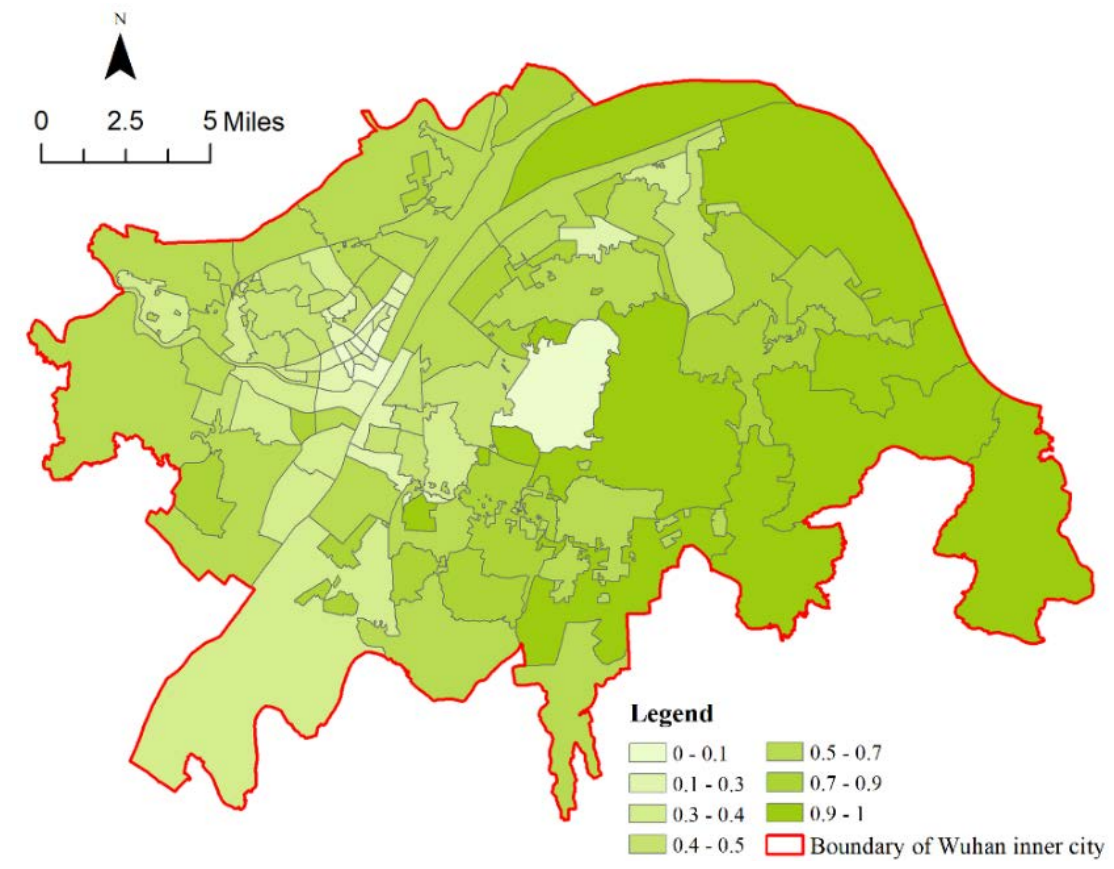

Figure 5: The percentage of urban green space within a buffer distance of $1 \mathrm{~km}$ (without water). 


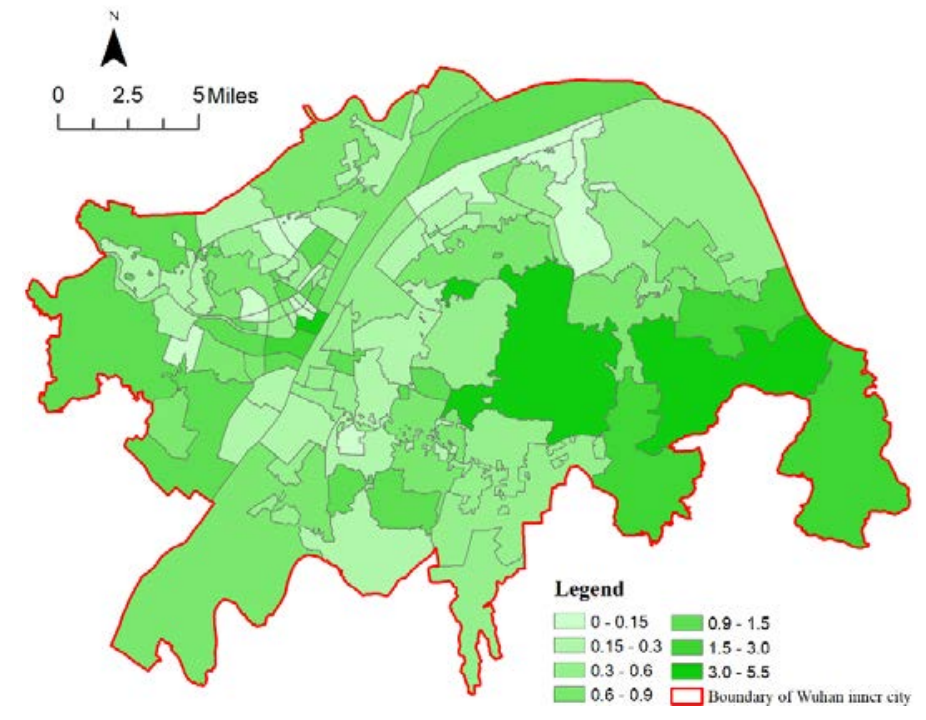

Figure 6: The ratio of arbor to shrub within a buffer distance of $1 \mathrm{~km}$.

in China. Yet, from the perspective of urban green space planning, we may attribute these issues to three dimensions. The first one is about the planning framework. In China, urban green space planning has been for such a long time regarded as a theme planning guided by the "top-down" approach. It means that urban green space planning is subordinate to master planning and should absolutely conform to it. So, what the central business urban green space planning has to deal with is to give more details to the pre-proposed "green space plan" by the related superordinate plans. Hence, as an echo to the idea of "garden city", "forest city", and "sponge city", to embody the ecological value rather than health benefits of urban green space is the key in its planning.

The second dimension, as a result of the first dimension, pertains to the indicators being applied in urban green space planning. As ecological value is the key, the amount of greenness is always the key in planning practice. So, there are almost no health-oriented indicators in the index system of urban green space planning and as a result residents' real demand of and access to urban green space has seldom been seriously considered. This further leads to the fact that availability rather than accessibility is emphasized in the index system even though accessibility is more or at least equally crucial for health-promotion [34]. So, it is not surprising to see that most Jiedaos have poor accessibility to and quality of urban green space.

The last one is related to the process and content of urban green space planning. Currently, urban green space planning is not treated as an incremental process but as a one-off work to draw a static blueprint. The central task is to define urban green space's overall function, to map its boundaries, and to detail the allocation of different types of green space (such as buffer green space and public green space). Inevitably, the dynamic characteristics of the green space and its interactions with urban development cannot be captured in the plans [35]. As a result, issues, such as management and improvement of the urban green space, its effects on the involved residents and local area, are always ignored, which might explain why green space with low quality and accessibility level tend to concentrate in some old areas in the inner city of Wuhan. 


\section{CONCLUSION}

Through five indicators, this paper evaluates the spatial distribution of urban green space in Wuhan inner city area by taking Jiedao as the analysis unit. The results reveal that from the perspective of health-promotion, its spatial distribution is relatively even in a general sense. Yet, if we consider residents' real demand of and accessibility to green spaces, we can see that most Jiedaos, especially those featured by old communities/factories have very low performance in both availability and accessibility. In addition, the quality of urban green space is also not satisfactory in many Jiedaos around the center of the inner-city area. The underlying causes to these issues from planning perspective, as has been explained, can be summarized into three key dimensions: urban green space's subordinate role in the ecologyorientation planning system; overlook or even ignorance of the health-promotion indicators in the planning index system; and a static view rather than an incremental view of the planning process.

Therefore, for health-promotion purpose, it is vital to shift from the ecology-centered value-orientation to a new one with both ecological and health values equally pursued. This will be the foundation for the establishment and application of the health-oriented indicators to be integrated into the planning index system; This shift also entails more attention to be paid to the users' socio-economic status and daily behavior. So, both the "top-down" and "bottom-up" approaches are needed so as to encourage public participation and promote the integrity of different levels of plans. Thus, the changing demands from residents can be identified in time and health-promotion can be equally emphasized in each planning step so as to keep urban green space function effectively for public health.

For a better development of this research, three points pertaining to the method need further effort. First, as the maps of NDVI and PGS are produced based upon the remote sensing image with a resolution of $30 \mathrm{~m} \times 30 \mathrm{~m}$, inevitably green space smaller than $900 \mathrm{~m}^{2}$ cannot be captured when calculating the relevant indicators. So, in Jiedaos, especially in the old ones where high density of construction and fragmented greens are more common to see, the calculated values might be lower than the real value. Whether it is true or not may need further on-site investigation or application of a higher resolution remote sensing map. Second, to evaluate availability and accessibility, the residence locations are supposed to be the centroids of the Jiedaos, which can cause inaccuracy of the evaluation. So, in order solve this problem, it is crucial to get the full map of all the residential communities in the study area which might be obtained by mining the mobile signaling data in Wuhan. Third, for each of the three dimensions (availability, accessibility and self-feature), only one indicator is applied. This in deed cannot reflect the full landscape of each dimension. So, what we need to do the next step is to propose and apply a more reasonable and comprehensive but succinct indicator set to represent each dimension in the evaluation.

\section{ACKNOWLEDGEMENTS}

This work is supported by the Science and Technology Department of Hubei Province under Grant (number 2017ADC073); China Thousands Talents Program under Grant (number D1218006).

\section{REFERENCES}

[1] van den Bosch, M. \& Sang, A.O., Urban natural environments as nature-based solutions for improved public health-A systematic review of reviews. Environmental Research, 158, pp. 373-384, 2017. DOI: 10.1016/j.envres.2017.05.040. 
[2] Kabisch, N., Qureshi, S. \& Haase, D., Human-environment interactions in urban green spaces: A systematic review of contemporary issues and prospects for future research. Environmental Impact Assessment Review, 50, pp. 25-34, 2015.

DOI: 10.1016/j.eiar.2014.08.007.

[3] Byomkesh, T., Nakagoshi, N. \& Dewan, A.M., Urbanization and green space dynamics in Greater Dhaka, Bangladesh. Landscape and Ecological Engineering, 8(1), pp. 45-58, 2012. DOI: 10.1007/s11355-010-0147-7.

[4] WHO, WHO methods and data sources for country-level causes of death 2000-2016. http://terrance.who.int/mediacentre/data/ghe/GlobalCOD_method_2000_2016.pdf?ua $=1$. Accessed on: 15 May 2018 .

[5] WHO, WHO launches Global Action Plan on Physical Activity. www.who.int/newsroom/detail/04-06-2018-who-launches-global-action-plan-on-physical-activity. Accessed on: 4 Jun. 2018.

[6] Huang, X.M. \& Wu, L.L., Spatial structure of urban greenbelt landscape in Nanjing city based on RS and GIS. Journal of Guangxi Teachers Education University (Natural Science Edition), 35(1), pp. 112-119, 2018.

[7] Yan, J.X., Zhang, J.F. \& Han, Y., Fragstats based study of urban green space landscape pattern in Handan city. Journal of Chinese Urban Forestry, 16(1), pp. 21-24, 2018.

[8] Hu, Z., Liebens, J. \& Rao, K.R., Linking stroke mortality with air pollution, income, and greenness in northwest Florida: An ecological geographical study. International Journal of Health Geographics, 7(1), p. 20, 2008. DOI: 10.1186/1476-072x-7-20.

[9] Zhang, L. \& Liu, Z.S., Optimization of park green space pattern in Changchun based on reachability. Acta Agriculturae Jiangxi, 30(3), pp. 59-63, 2018.

[10] Li, W.N. et al., Study on accessibility of square and park with greenbelt in center city. Geomatics and Spatial Information Technology, 41(4), pp. 94-97, 2018.

[11] Hystad, P. et al., Residential greenness and birth outcomes: Evaluating the influence of spatially correlated built-environment factors. Environmental Health Perspectives, 122(10), pp. 1095-1102, 2014. DOI: 10.1289/ehp.1308049.

[12] Markevych, I. et al., A cross-sectional analysis of the effects of residential greenness on blood pressure in 10-year old children: results from the GINIplus and LISAplus studies. BMC Public Health, 14(1), p. 477, 2014. DOI: 10.1186/1471-2458-14-477.

[13] Maas, J., Verheij, R.A., Groenewegen, P.P., de Vries, S. \& Spreeuwenberg, P., Green space, urbanity, and health: how strong is the relation? Journal of Epidemiology and Community Health, 60(7), pp. 587-592, 2006. DOI: 10.1136/jech.2005.043125.

[14] Maas, J., van Dillen, S.M.E., Verhei, R.A. \& Groenewegen, P.P., Social contacts as a possible mechanism behind the relation between green space and health. Health and Place, 15(2), pp. 58-595, 2009. DOI: 10.1016/j.healthplace.2008.09.006.

[15] Storgaard, R.L., Hansen, H.S., Aadahl, M. \& Glümer, C., Association between neighbourhood green space and sedentary leisure time in a Danish population. Scandinavian Journal of Public Health, 41(8), pp. 846-852, 2013.

DOI: $10.1177 / 1403494813499459$.

[16] Almanza, E., Jerrett, M., Dunton, G., Seto, E. \& Pentz, M.A., A study of community design, greenness, and physical activity in children using satellite, GPS and accelerometer data. Health and Place, 18(1), pp. 46-54, 2012.

DOI: 10.1016/j.healthplace.2011.09.003.

[17] Grigsby-Toussaint, D.S., Chi, S.-H. \& Fiese, B.H., Where they live, how they play: Neighborhood greenness and outdoor physical activity among preschoolers. International Journal of Health Geographics, 10(1), p. 66, 2011. DOI: 10.1186/1476072x-10-66. 
[18] Van Herzele, A. \& de Vries, S., Linking green space to health: a comparative study of two urban neighbourhoods in Ghent, Belgium. Population and Environment, 34(2), pp. 171-193, 2012. DOI: 10.1007/s11111-011-0153-1.

[19] Zhang, W.J., Yang, J., Ma, L. \& Huang, C., Factors affecting the use of urban green spaces for physical activities: Views of young urban residents in Beijing. Urban Forestry and Urban Greening, 14(4), pp. 851-857, 2015.

DOI: 10.1016/j.ufug.2015.08.006.

[20] Schipperijn, J., Bentsen, P., Troelsen, J., Toftager, M. \& Stigsdotter, U.K., Associations between physical activity and characteristics of urban green space. Urban Forestry and Urban Greening, 12(1), pp. 109-116, 2013.

DOI: 10.1016/j.ufug.2012.12.002.

[21] Barton, J. \& Pretty, J., What is the best dose of nature and green exercise for improving mental health? A Multi-Study Analysis, 44(10), pp. 3947-3955, 2010.

[22] Health Survey in Wuhan. http://hb.sina.com.cn/news/b/2017-04-20/detail-ifyepsch 1854190.shtml. Accessed on: 23 May 2018.

[23] 'Healthy Wuhan 2030' Plan Compendium (consulation version). www.qiaokou.gov.cn/xxgk/xxgkml/zfxxgk/ghjh_442/zxgh/201709/t20170911_1919 43.shtml. Accessed on: 23 May 2018.

[24] 'Healthy Wuhan 2030' Action Plan (consulation version). www.whwsjs.gov.cn/front/ web/showDetail/1500449284198. Accessed on: 23 May 2018.

[25] National sport field area being 1.57 square meter per capita in 2016. www.xinhuanet.com/sports/2016-04/07/c_1118559597.htm. Accessed on: 23 May 2018.

[26] Warburton, D.E.R., Nicol, C.W. \& Bredin, S.S.D., Health benefits of physical activity: The evidence. Canadian Medical Association Journal, 174(6), pp. 801-809. DOI: $10.1503 / \mathrm{cmaj} .051351$.

[27] Zhu, Z.Y. \& Wu, S.Q., Street-landscape survey in Beijing. Journal of Chinese Landscape Architecture, 11(1), pp. 37-44, 1995.

[28] Zhang, X.W. et al., Analysis of the ratio of arbor to shrub of several types of green space in Beijing. Journal of Beijing Forestry University, S1, pp. 183-188, 2010.

[29] Rui, Y. et al., The spatio-temporal evolutionary characteristics and the impact mechanism of national garden cities in China. Geographical Research, 1, pp. 20-36, 2018.

[30] Zhu, Q.F., Li, Z. \& Yang, X., An improvement of evaluating method on tourist concentration degree with geographic concentration index. Tourism Tribune, 26(4), pp. 26-29, 2011.

[31] Wu, L.F., Research on the Equity of Regional Distribution and the Influencing Factors of Doctors' Human Resources in Shanghai. Shanghai Academy of Social Sciences: China, 2017.

[32] Maas, J., Verheij, R.A., de Vries, S., Spreeuwenberg, P., Schellevis, F.G. \& Groenewegen, P.P., Morbidity is related to a green living environment. Journal of Epidemiology and Community Health, 63(12), pp. 967-973, 2009.

DOI: $10.1136 /$ jech.2008.079038.

[33] Liu, G.C., Wilson, J.S., Qi, R. \& Ying, J., Green neighborhoods, food retail and childhood overweight: differences by population density. American Journal of Health Promotion, 21(4), pp. 317-325, 2007. DOI: 10.4278/0890-1171-21.4s.317.

[34] WHO, Urban green space and health: A review of evidence, 2016.

[35] Wen, Q.P., Discussions on green space system planning in urban and rural coordination context. Landscape Architecture, 6, pp. 144-148, 2013. 